\title{
Fracture and Size Effect on Strength of Plain Concrete Disks under Biaxial Flexure Analyzed by Microplane Model M7
}

\author{
Kedar Kirane'; Zdeněk P. Bažant, P.E., Hon.M.ASCE²; and Goangseup Zi
}

\begin{abstract}
The biaxial tensile strength of concrete (and ceramics) can be easily tested by flexure of unreinforced circular disks. A recent experimental study demonstrated that, similar to plain concrete beams, the flexural strength of disks suffers from a significant size effect. However, the experiments did not suffice to determine the size effect type conclusively. The purpose of this study is to use three-dimensional stochastic finite-element analysis to determine the size effect type and shed more light on the fracture behavior. A finite-element code using the microplane constitutive Model M7 is verified and calibrated by fitting the previously measured load-deflections curves and fracture patterns of disks of thicknesses 30, 48, and $75 \mathrm{~mm}$, similar in three dimensions, and on flexure tests on four-point loaded beams. It is found that the deformability of the supports and their lifting and sliding has a large effect on the simulations, especially on the fracture pattern, and the strength and Young's modulus of concrete must be treated as autocorrelated random fields. The calibrated model is then used to analyze the size effect over a much broader range of disk thicknesses ranging from 20 to $192 \mathrm{~mm}$. The disks are shown to exhibit the typical energetic size effect of Type I, that is, the disks fail (under load control) as soon as the macrofracture initiates from the smooth bottom surface. The curve of nominal strength versus size has a positive curvature and its deterministic part terminates with a horizontal asymptote. The fact that material randomness had to be introduced to fit the fracture patterns confirms that the Type 1 size effect must terminate at very large sizes with a Weibull statistical asymptote, although the disks analyzed are not large enough to discern it. DOI: 10.1061/(ASCE)EM.1943-7889.0000683. @ 2014 American Society of Civil Engineers.
\end{abstract}

Author keywords: Biaxial test; Flexural strength; Damage; Fracture; Size effect; Concrete; Finite-element method (FEM); Stochastic models.

\section{Introduction}

In many structures, such as road pavements, airplane runways, dams, and nuclear containments, concrete is subjected to biaxial tension, and the biaxial strength needs to be known (Mindess and Francis 1981; Lee et al. 2004). However, in a quasi-brittle material, such as concrete, a size effect on the strength must be expected. For uniaxial tension, for example in flexure of unreinforced beams, a strong size effect is well documented (Bažant and Novák 2000, 2001). For biaxial tension in flexure of unreinforced plates, a strong size effect has been demonstrated by Zi et al. (2008, 2013). However, the size range of these tests was limited; consequently, the type of size effect could not be determined conclusively. The purpose of this study is to use finite-element simulations to extend the size range and unequivocally ascertain the type of size effect.

Ascertaining the type of size effect is important for large structures, such as thick pavements, because the large-size asymptotic behaviors of Type I and Type II size effects are very different. Although the Type II size effect is purely energetic and has an

\footnotetext{
${ }^{1}$ Graduate Research Assistant, Dept. of Mechanical Engineering, Northwestern Univ., 2145 Sheridan Rd., A127, Evanston, IL 60208.

${ }^{2}$ McCormick Institute Professor and W. P. Murphy Professor, Dept. of Civil and Mechanical Engineering and Materials Science, Northwestern Univ., 2145 Sheridan Rd., A132, Evanston, IL 60208 (corresponding author). E-mail: z-bazant@northwestern.edu

${ }^{3}$ Professor, School of Civil, Environmental and Architectural Engineering, Korea Univ., Seoul 136-701, Republic of Korea.

Note. This manuscript was submitted on February 25, 2013; approved on June 24, 2013; published online on June 26, 2013. Discussion period open until August 1, 2014; separate discussions must be submitted for individual papers. This paper is part of the Journal of Engineering Mechanics, Vol. 140, No. 3, March 1, 2014. (OASCE, ISSN 0733-9399/2014/3-604-613/ $\$ 25.00$.
}

asymptote of a downward slope of $-1 / 2$ [in agreement with linear elastic fracture mechanics (LEFM)], the Type I is a combined energetic-statistical size effect, which has a straight-line statistical asymptote of a much smaller downward slope equal to $-n / m$ where $m$ is the Weibull modulus (about 24 for concrete), and $n=3$ is the number of spatial dimensions (Bažant and Novák 2000).

A simple way to measure the biaxial strength of a brittle material is the flexural test of a disk pioneered by Zi et al. (2008). In Zi's test, the disk is supported along its perimeter on a circular ring and is loaded at the center by a small circular ring shown in Fig. 1. This is analogous to the four-point bending test of a beam. The strength measured is the equibiaxial strength. This testing procedure is common for ceramics (Morrell 1998), and the elastic-stress distribution in the disk is axisymmetric and well known (Fessler and Fricker 1984). A standard test for concrete exists [ASTM C1550 test (ASTM 2008)] in which the elastic field is threefold symmetric. In this test, the disk is supported on three pivots along the perimeter and is subjected to a concentrated load at the center. The stress is equibiaxial only at the load point. This test will not be considered here.

The fracture patterns obtained by $\mathrm{Zi}$ et al. (2013) and Kim et al. (2012) in their biaxial flexure tests of various sizes are shown in Fig. 2. Although the loading and the elastic-stress state in these tests are axisymmetric, the fracture pattern is not. Matching fracture patterns is a challenging but important check on the correctness of numerical simulations. Aside from analyzing the observed size effect, the current study will also focus on reproducing these fracture patterns.

The material model chosen for this purpose is the microplane model. This is a semimultiscale model because it captures only interaction among the defects on planes of various orientations within the material; however, it does not capture interactions at a distance because the inelastic phenomena are lumped into one point of the macroscopic continuum. A conceptual advantage is that the 
constitutive law is expressed in a vectorial form rather than a tensorial form (Bažant and Oh 1985). The microplane model has been proven capable of accurately capturing the damage, fracture behavior, and size effect in various quasi-brittle materials, which exhibit postpeak softening damage because of distributed cracking (Bažant et al. 2000; Caner and Bažant 2013).

In this study, the microplane model is first calibrated and validated by fitting previously obtained experimental data ( $\mathrm{Zi}$ et al. 2013) on the loads, strains, and deflections of disks of three sizes, and by matching the fracture patterns. The effects of deformable supports and material randomness are also analyzed. Finally, the calibrated model is used to predict the size effect for a greatly extended size range, which permits determining the size effect type, which turns out to be the same as assumed in the previous experimental study.

\section{Numerical Simulation of Biaxial Flexure Tests}

\section{Microplane Model M7}

The microplane Model M7 is the latest version in a series of progressively improved microplane models labeled M0-M6, which were developed primarily for concrete (Bažant and Oh 1985). Other microplane models have been developed for clays, soils,

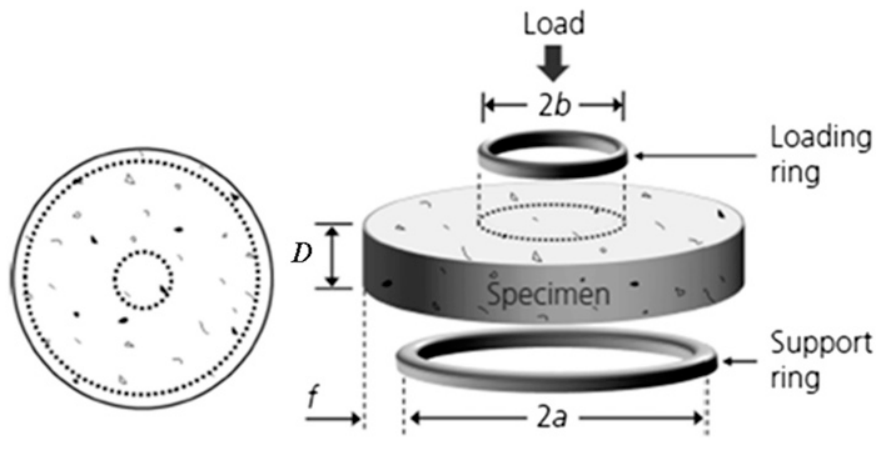

Fig. 1. Schematic of the biaxial flexure test method rocks, rigid foams, clays, shape memory alloys, annulus fibrosus, and composites (prepreg laminates and braided) (Bažant et al. 2000; Caner and Bažant 2000; Cusatis et al. 2008; Caner et al. 2011). The microplane model, supplemented by some localization limiter with material characteristic length, has been proven to give rather realistic predictions of the constitutive and damage behavior of quasi-brittle materials over a broad range of loading scenarios, including uniaxial, biaxial, and triaxial loadings with postpeak softening, compression-tension load cycles, opening and mixedmode fractures, tension-shear failure, and axial compression followed by torsion.

The basic idea of the microplane model is to express the constitutive law not in terms of tensors but in terms of the vectors of stress and strain acting on a generic plane of any orientation in the material microstructure, which is called the microplane. Considering all such planes captures interaction between various orientations, which makes the model semimultiscale in nature (a fully multiscale model must also capture interactions at a distance).

The use of vectors was inspired by an idea of Taylor (1938) and has been used extensively in Taylor models for plasticity of polycrystalline metals (Batdorf and Budianski 1949; Asaro and Rice 1977). However, there are important conceptual differences. First, to avoid model instability in postpeak softening, a static constraint of the microplanes had to be replaced by a kinematic one (Bažant 1984; Bažant and Oh 1985). Therefore, the strain (rather than stress) vector on each microplane is the projection of the macroscopic strain (rather than stress) tensor. Second, a variational principle (principle of virtual work) was introduced to relate the stresses on the microplanes of all possible orientations to the macrocontinuum stress tensor and ensure equilibrium. These features made possible simulation of strain softening damage because of, for example, multidirectional distributed tensile cracking (for details, see Bažant et al 2000; Caner and Bažant 2000; Cusatis et al 2008; Caner et al 2011).

\section{Calibration and Validation of the Microplane Model}

Microplane Model M7 was incorporated in the commercial finiteelement code ABAQUS 6.11 using the explicit user-defined material routine VUMAT (ABAQUS 2011). Then, the M7 free parameters were calibrated by fitting the biaxial flexure test results of $\mathrm{Zi}$ et al. (2013). Three disks with the size ratio of 1:1.6:2.5 were

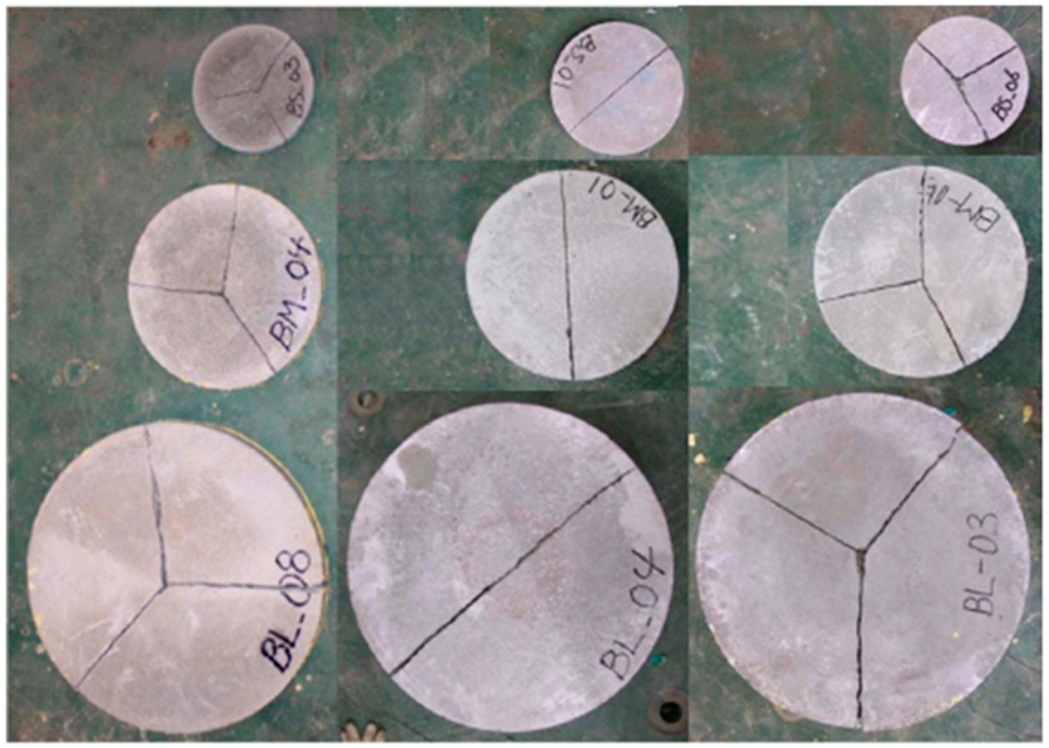

Fig. 2. Experimentally observed fracture patterns in concrete disks of three sizes during the biaxial flexure tests 
tested. The disks were geometrically similar in three dimensions, that is, the disks and the radii of the support and loading ring were scaled in the same ratio (Table 1).

To prevent spurious mesh sensitivity, it is essential to use the microplane model in conjunction with some localization limiter (Črvenka et al. 2005). Here, the modeling is performed in the sense of the crack band model, in which the bandwidth $w_{c}$ is a material property, in this case estimated as $13 \mathrm{~mm}(0.51 \mathrm{in}$.) [which is twice the maximum aggregate size of $6.5 \mathrm{~mm}(0.255 \mathrm{in}$.) ]. The damage is assumed to localize into a band of this width. Although changing the element size is possible, the crack band model is most accurate when the element size is kept equal to $w_{c}$. Therefore, the disks were meshed by four-noded tetrahedral elements (C3D4) elements of average size, $w_{c}=13 \mathrm{~mm}(0.51 \mathrm{in}$.). The mesh was obtained by random Voronoi tessellation, which is necessary to avoid directional bias of crack propagation. To speed up the explicit dynamic integration, the natural time scale, irrelevant to statics, was contracted by mass scaling, which involves artificially scaling the density of the material. A scaling factor of 1,000 was chosen. It was checked that higher factors would give the same results but would produce some numerical noise. Furthermore, although the loading rate used in the tests was $1 \mathrm{~mm} / \mathrm{min}(0.0393 \mathrm{in} . / \mathrm{min})$, the rate used in the numerical simulations was higher, equal to $6 \mathrm{~mm} / \mathrm{min}(0.2362 \mathrm{in} . / \mathrm{min})$, which helped to avoid excessive running times. The change of rate was justified because a rate-independent form of M7 was used, and the inertia forces were checked to be negligible.

In the tests, the strain evolutions in gauges mounted roughly at the disk center on the bottom surface were measured (Kim et al. 2012). To calibrate and validate the model, the load-strain plots for disks of three sizes were optimally fitted using the measured Young's modulus $E$ and compressive strength $f_{c}^{\prime}$ and searching for the best value of the radial scaling parameter $k_{1}$ of the microplane model; see the fits in Fig. 3. All the other adjustable microplane parameters were taken at their default values (Caner and Bažant 2013) (Table 2). The average logarithmic strain in the radial direction was determined for the elements located roughly at the bottom at the disk center. This average strain was plotted against the total reaction.

Fig. 3 compares the test data with the numerical results for the disks of all three sizes. The dotted lines represent the test data whereas the solid lines represent the prediction using the model. The agreement is seen to be quite close. The microplane model parameters were adjusted to match the mean response of the specimen. The range of scatter in the strength values observed in the tests is shown by error bars in the plot, and the mean value is marked on each error bar by a dash. The load-strain plot was not measured for all of the specimens tested for strength. Having shown the microplane model to be capable of predicting the response of biaxially bent disks, the size effect and fracture can now be analyzed.

\section{Size Effect in the Disks}

The size effect is an important consideration for all quasi-brittle materials (Bažant and Planas 1998). It has been thoroughly investigated

Table 1. Dimensions of the Disks Used for Biaxial Flexure Testing

\begin{tabular}{lccc}
\hline Dimension $(\mathrm{mm})$ & Small disk & Medium disk & Large disk \\
\hline Thickness & 30 & 48 & 75 \\
Radius & 131 & 210 & 328.5 \\
Radius of support ring & 125 & 200 & 312.5 \\
Radius of loading ring & 31.5 & 50 & 78 \\
\hline
\end{tabular}

for specimens with uniaxial stress, but tests with biaxial stress were lacking until the work of $\mathrm{Zi}$ et al. (2013).

\section{Size Effect in Biaxial Strength}

For axisymmetrically loaded circular disks, the biaxial flexural strength at the center bottom is, according to elasticity (Timoshenko and Woinowsky-Krieger 1959)

$$
f_{t}=c_{u} \frac{P_{\max }}{D^{2}}, \quad c_{u}=\frac{3}{4 \pi}\left[2(1+v) \ln \left(\frac{a}{b}\right)+\frac{(1-v)\left(a^{2}-b^{2}\right)}{R^{2}}\right]
$$

where $P_{\max }=$ peak load; $c_{u}=$ dimensionless geometry factor; $R$ $=$ radius of the disk; $a=$ radius of the support ring; $b=$ radius of the loading ring; $D=$ thickness of the disk; and $v=$ Poisson's ratio.

The biaxial strengths calculated from the peak loads predicted for various disk sizes by the finite-element code with M7 are compared in Fig. 4 with the values obtained from the measured peak loads. The strength values from individual tests are shown by crosses $(\times)$, and their mean is shown by solid dots. The M7 predictions match the

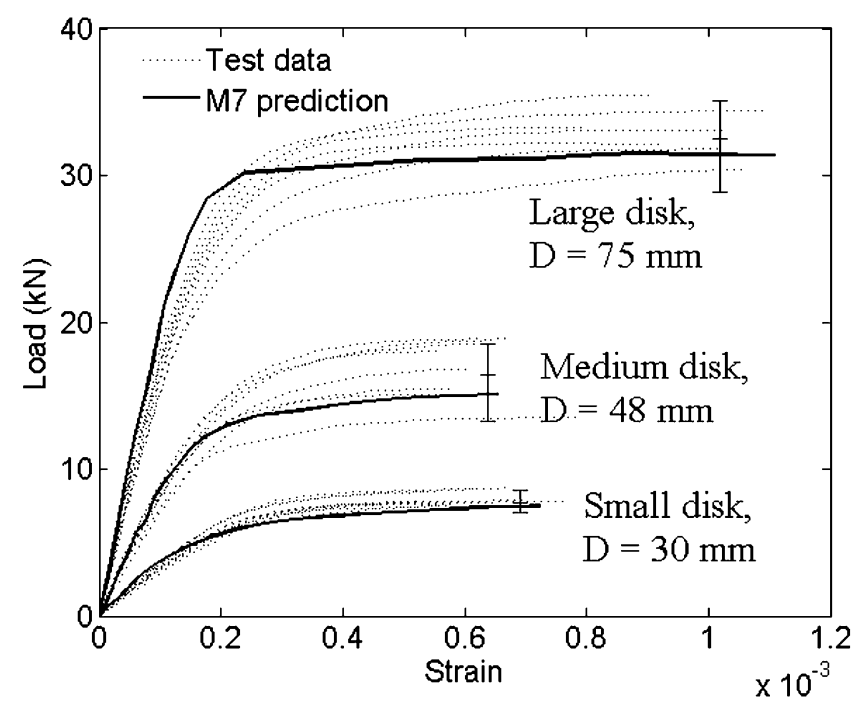

Fig. 3. Comparison of load-strain curves from biaxial flexure experiments and predictions using the microplane Model M7

Table 2. Elastic Material Constants and Parameters of the Microplane Model M7

\begin{tabular}{lc}
\hline Material parameter (unit) & Value \\
\hline Young's modulus $E(\mathrm{MPa})($ mean$)$ & 27,264 \\
Poisson's ratio & 0.22 \\
Compressive strength $f_{c}^{\prime}(28$ days) $(\mathrm{MPa})$ & 33 \\
Density $\left(\mathrm{kg} / \mathrm{m}^{3}\right)$ & 2,800 \\
Radial scaling parameter $k_{1}$ & $1.05 \times 10^{-4}$ \\
$k_{2}$ & 110 \\
$k_{3}$ & 30 \\
$k_{4}$ & 100 \\
$k_{5}$ & 1 \\
$k_{6}$ & $1 \times 10^{-4}$ \\
$k_{7}$ & 1.8 \\
\hline
\end{tabular}


mean values of the test data quite well. However, the size effect curve from both the simulations and tests is almost straight within the size range of the tests. Because the size range is too narrow, one cannot conclude from the test data alone whether the size effect is Type 1 (positive curvature) or Type 2 (negative curvature).

To answer the question of size effect type, the M7 model calibrated by test data has been used to compute the peak loads for geometrically scaled specimens of three additional sizes [thicknesses $D=20,120$, and $192 \mathrm{~mm}(0.7874,4.7244$, and 7.559 in.)]. To avoid the approximations and complexity of the crack band model adjustment when the element size is increased, it was preferred to retain the same element size across all models. Therefore, the finiteelement models for the largest size disks turned out to be very big. The largest disk had close to 1 million elements and $\sim 175,000$ nodes. The large-scale simulations were performed using parallel computing on Quest (Northwestern University Information Technology 2012), which is a high-performance computing (HPC) cluster system at Northwestern University. A summary of the finiteelement models is shown in Table 3. The results are shown again in Fig. 4. With the addition of these data points shown, it now becomes clear that the size effect is Type 1 (Bažant and Planas 1998). Furthermore, the computed data show that the size effect plot reaches an asymptotic bound when the thickness $D$ is about $120 \mathrm{~mm}$.

In this regard, the asymptotic bound exists only if the material is considered to be deterministic. In reality, the tensile strength of concrete is random; in that case, the Type 1 size effect curve terminates with the Weibull power-law size effect, which in Fig. 4(a) would represent the straight-line asymptote of a downward slope equal to $-n / m$ where $m$ is the Weibull modulus (Bažant and Novák 2000) (about 24) and $n$ is the number of spatial dimensions.

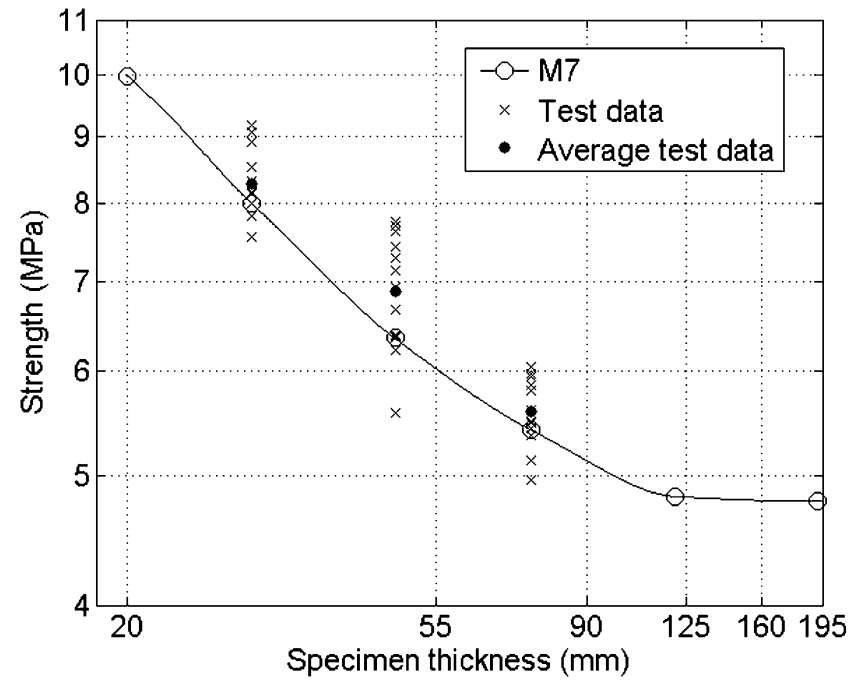

Fig. 4. Comparison of biaxial size effect from biaxial flexure experiments and predictions using the microplane model M7

Table 3. Details of the Finite-Element Models of Disks of Various Sizes

\begin{tabular}{lcc}
\hline Thickness $D(\mathrm{~mm})$ & Number of nodes & Number of elements \\
\hline 20 & 656 & 2,357 \\
30 & 1,763 & 7,433 \\
48 & 5,793 & 27,531 \\
75 & 16,010 & 80,927 \\
120 & 52,644 & 279,168 \\
192 & 174,921 & 958,174 \\
\hline
\end{tabular}

However, just like concrete beam flexure, this statistical part of the size effect would be important only for very thick slabs, for which no data exist yet. The basic feature of Type 1 size effects is that a macroscopic continuous crack initiates from a smooth (unnotched) surface only during postpeak softening. The finite-element results confirm this feature for all the disk sizes. They show that, before reaching the peak load, there are no elements with zero stress and only few ones with very low stress (less than $0.5 \mathrm{MPa}$ ).

\section{Comparison with the Size Effect on Uniaxial Strength in Beam Flexure}

For better verification and calibration of Model M7, it is useful to make a comparison with the size effect on uniaxial flexural strength. $\mathrm{Zi}$ et al. (2013) reported the results of such tests on four-point bent beams. They observed the biaxial flexural strength of concrete to be higher than the uniaxial flexural strength. This is in agreement with the Griffith flaw theory (Mindess and Francis 1981) according to which a second tensile principal stress at right angles gives a higher strength for open flaws. However, there have also been reports of contrary results indicating the biaxial tensile strength to be lower than the uniaxial tensile strength, for example for dense alumina ceramics (Giovan and Sines 1979). But these tests were made on fine-grained ceramics (with grain size $20-30 \mu \mathrm{m}$ ), for which the deterministic size effect is, at normal laboratory scale, nil and only the Weibull statistical size effect operates. These contrary results have been supported by Batdorf statistics (Batdorf and Crose 1974) according to which the biaxial tension gives a higher failure probability than the uniaxial tension.

To clarify this issue and to provide additional validation, the fourpoint bend tests of biaxial flexural strength of beams of three sizes, which were performed by $\mathrm{Zi}$ et al. (2013), were also simulated with M7. The same element size and the same calibrated material parameters were retained. The uniaxial nominal strengths, $f_{t}$, were obtained from the peak loads as follows:

$$
f_{t}=c_{u} \frac{P_{\max }}{b D}, \quad c_{u}=\frac{L}{D}
$$

where $P_{\max }=$ peak load; $L=$ span length of the beam; $b=$ depth of the beam; and $D=$ thickness of the beam, which is used as the

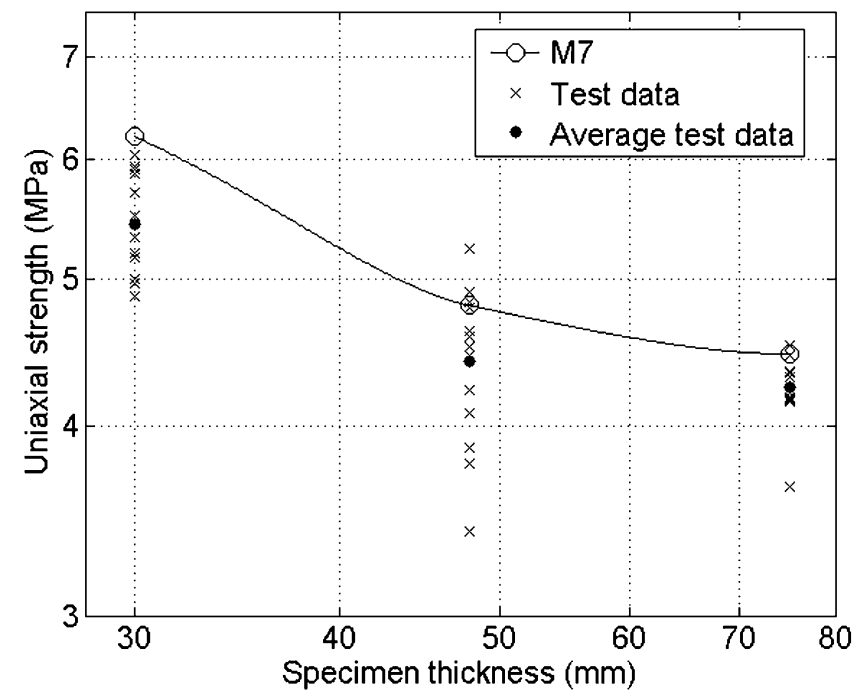

Fig. 5. Comparison of uniaxial size effect from four-point bend experiments and predictions using the microplane Model M7 
characteristic size. The strength values are plotted against size $D$ and compared with the test results as shown in Fig. 5. Again, the strength values from individual tests are shown by crosses, and their mean is shown by solid dots. The predicted uniaxial strength values are in good agreement with the mean strength values from the test. The predicted uniaxial strength of the small beam is a bit higher than the experimental mean value. One possible explanation is inevitable random error of experiments. Another possible explanation might be that, because of a fixed mesh size of $13 \mathrm{~mm}$, the mesh for the small beam $(D=30 \mathrm{~mm})$ is too coarse, which would cause a slightly stiffer response. The agreement for the medium and large sizes is satisfactory. The predicted uniaxial flexural strength values are indeed lower than the biaxial flexural strength values and are consistent with the experiments. Both curves resemble a typical Type I size effect curve. This further validates the predictions of the microplane model.

\section{Results and Discussion of Fracture Patterns and Randomness}

\section{Simulations with Stiff Supports}

The initial simulations have used a simple support condition in which the loading was applied directly to the nodes on the top and bottom surfaces of the disks, which implies that separation of the disk from the support and the steel loading plate is not allowed. To visualize the localization of damage and fracture patterns, contours of the maximum principal strain on the bottom surface of the disk are plotted. Fig. 6(a) shows the fracture patterns obtained from the simulations for all three sizes. For each size, the damage localizes in multiple radial bands originating at the center. Five to six distinct radial crack bands are observed in each case. However, this is not what is seen in the tests.

The simulation was continued further into the postpeak softening regime for the small-size disks. The corresponding damage is shown in Fig. 6(b). The original crack bands that originated at the center are seen to bifurcate before reaching the edge. These fracture patterns are remarkably similar to those reported by Giovan and Sines (1979) and Shetty et al. (1983). However, they disagree with the present tests, which consisted of one diametrical crack or three radial cracks emanating from the center. Besides, the disks failed dynamically right after the peak load whereas, in these initial simulations, a stable postpeak softening could be observed.

\section{Simulations with Deformable Rings and Lifting from Supports}

It was suspected that the cause of these disagreements was the assumption of rigid connection at the supports. The supporting perimeter ring [of thickness $20 \mathrm{~mm}$ (0.7874 in.)] and the smaller loading ring under the steel platen were made of a flexible elastomer; the disk was not prevented from sliding and lifting from the supporting ring during fracture growth. Both phenomena suppressed the tendency to develop one diametric crack. Therefore, the supporting and loading rings are simulated by finite elements, both made of an elastomer with Young's modulus of about $50 \mathrm{MPa}(7.25 \mathrm{ksi})$ and a Poisson ratio's of 0.49 (Engineering Toolbox 2012). Very thin polytetrafluorethylene layers inserted between the rings and the concrete to minimize friction are simulated, too, considering a very low friction coefficient, which is equal to 0.03 .

With these refinements (Fig. 1), the simulations display rather different postpeak behavior and fracture patterns [Fig. 7(a)]. One

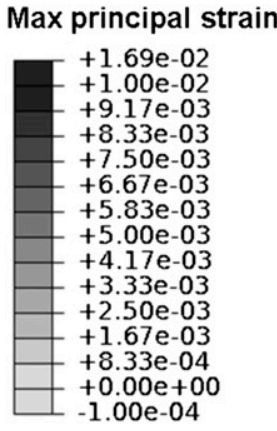

(a)

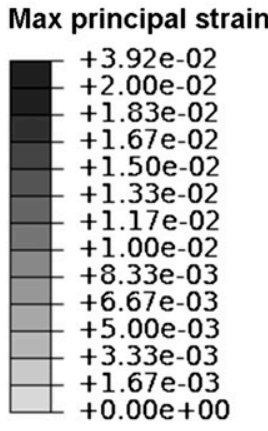

(b)
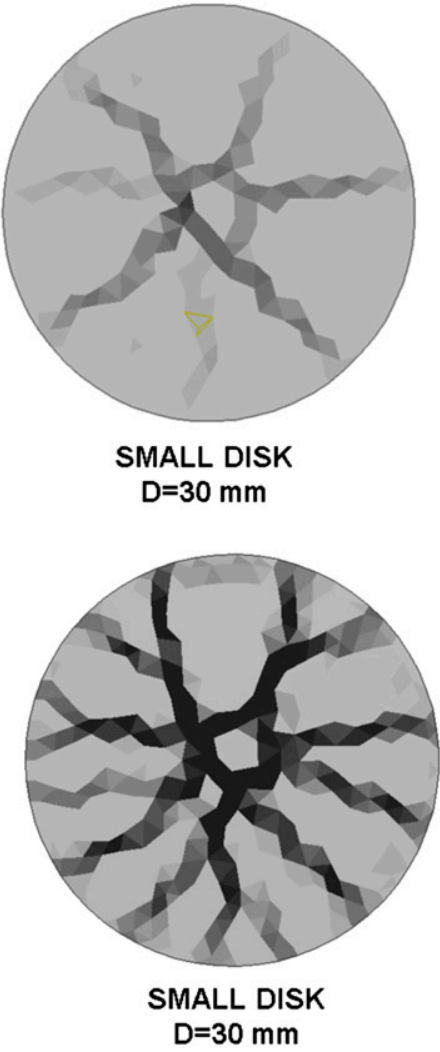
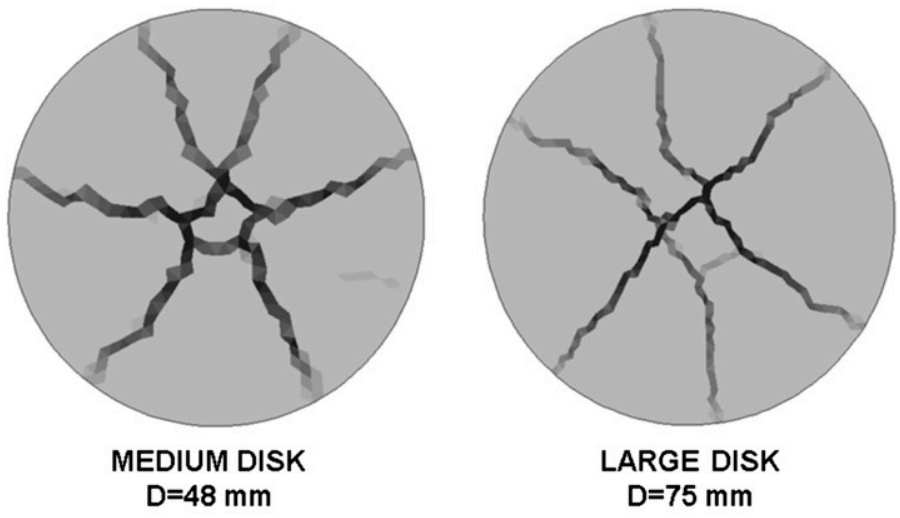

$\mathrm{D}=48 \mathrm{~mm}$

Fig. 6. (a) Predicted fracture patterns from simulations using an idealized test setup; (b) fracture pattern on the small-sized disk from a prolonged simulation using an idealized test setup 


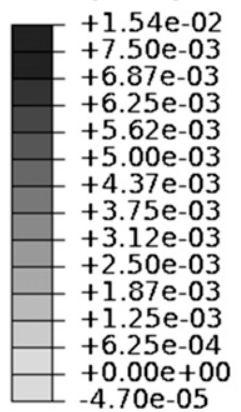

(a)

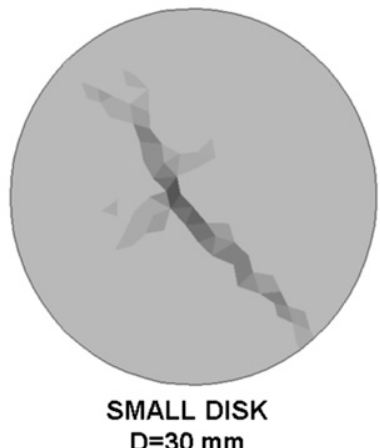

$\mathrm{D}=30 \mathrm{~mm}$

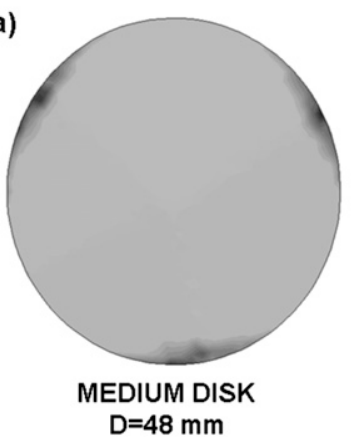

$$
\mathrm{D}=48 \mathrm{~mm}
$$
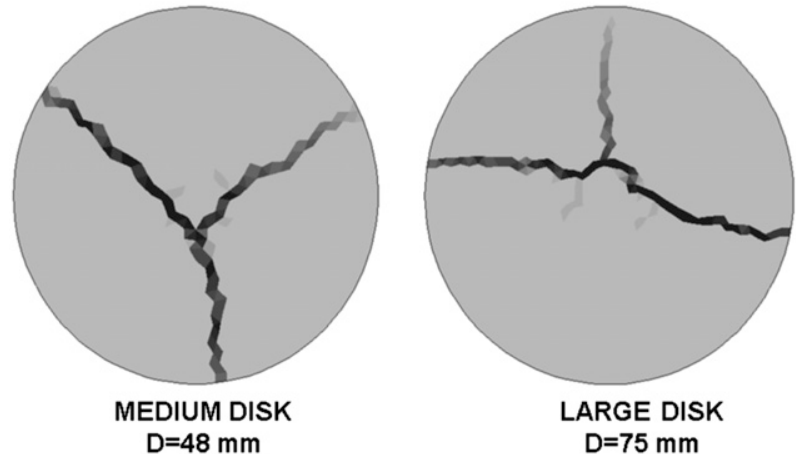

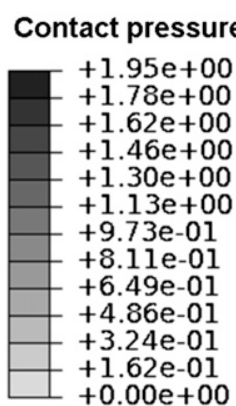

(b)

Fig. 7. (a) Predicted fracture patterns from simulations using a realistic test setup; (b) contour plot of contact pressure for the medium-size disk

diametric crack is now obtained for the small size, and three radial cracks are obtained for the medium and large sizes. Notably, the stability of equilibrium is lost after the peak load, and the dynamic snap-through, revealed by the nearly vertical drop of load in Fig. 8(b), indicates that a snap-back must occur on the equilibrium path.

This conclusion is further reinforced by the plot of kinetic energy versus time in Fig. 8(c), which shows a sudden spike in the kinetic energy precisely at the instant corresponding to the vertical drop in the load, and by the subsequent oscillations, which dissipate the kinetic energy (and are also noticeable in the load-deflection plots). Indeed, the kinetic energy $K$ acquired during the snap-through must come from somewhere, and it must in fact be exactly equal to the area $A$ between the snap-back equilibrium path and the vertical snapthrough; see the schematic plot in Fig. 8(d) (Bažant and Cedolin 2010). If there were no snap-back, the vertical stress drop could not be dynamic; it would have to be an equilibrium path, and only then area $A$ in Fig. 8(d) could be zero. For comparison, the loaddisplacement curves from simulations with rigid supports that show stable postpeak softening are shown in Fig. 8(a). Although the postpeak behavior is completely different, the peak load stays virtually unchanged, which is to be expected because the material properties have not changed [The initial slope in Fig. 8(b) is the effective stiffness of the disk in the series with the loading and support layer.]

A study of the postpeak equilibrium path with snap-back, which would require switching to an implicit integration algorithm with arc length control, is not the objective of this paper. The time instant of the instability and dynamic snap-through corresponds to the onset of this fracture pattern. This pattern agrees well with the observations. Also seen in Fig. 7(b) is a contour plot of various contact pressure values for the medium size. The locations with a high contact pressure exactly correspond to the locations where the damage is seen to localize. A zero contact pressure signifies the possibility of lift-off. In other words, although rigid contact of supports with the disk was seen to produce a conical axisymmetric deflection surface and stable postpeak softening with many radial cracks, a softly applied load gives a nonaxisymmetric surface, postpeak instability with sudden load drop, and a failure with either one diametric crack or three radial cracks, which is consistent with the experiments.

\section{Random Field of Concrete Properties and Its Autocorrelation}

Concrete stiffness and strength are highly random, and their distributions may have a significant effect, even on the mean response. So now, the Young's modulus $E$ is assumed to represent an autocorrelated field with Gaussian (normal) distribution. In the microplane model, the strength is an outcome of the specified tensile normal boundary. Because this boundary is a function of the Young's modulus (Caner and Bažant 2013), the strength is also represented as a random field. The autocorrelation length is initially considered to be equal to the maximum aggregate size $[13 \mathrm{~mm}$ ( $0.51 \mathrm{in}$.)], and the element size $h$ is chosen to be the same as mentioned previously. In that case, it suffices to assign each element a randomly generated $E$ value (in $A B A Q U S$, this is easily implemented in the user's material subroutine VUMAT). The randomly generated $E$ values follow a Gaussian distribution with a mean value of $E$, which is provided in Table 2 , and a coefficient of variation $\sim 0.05$. The truncated cumulative probability distribution of the $E$ values for the small-size disk is shown in Fig. 9. All the other settings remain unchanged.

Fig. 10(a) shows the simulated fracture patterns obtained for the simulations with random Young's modulus and strength. Once again, a sudden load drop (or a dynamic snap-through) is observed just after the peak. However, only one single diametric crack is now obtained for the small and medium sizes whereas three radial cracks are obtained for the large size. Therefore, by modeling the disk with 

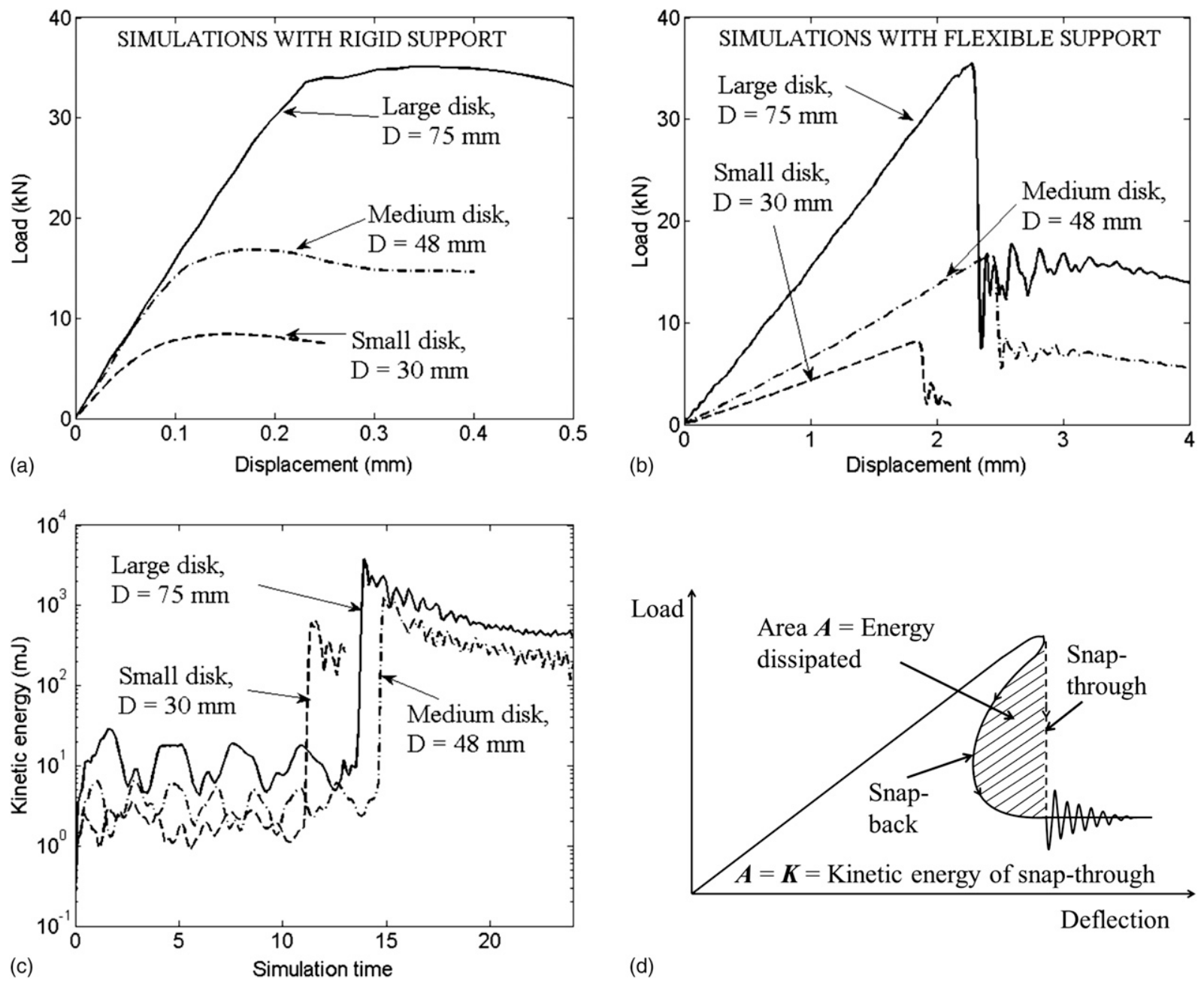

(d)

Fig. 8. (a) Load-displacement curves from simulations using stiff supports showing stable postpeak softening; (b) load-displacement curves from simulations using a realistic test setup showing an instability with a sudden load drop (or snap-through) right after peak load (implying the presence of snap-back on the equilibrium path); (c) kinetic energy versus time curve showing a sudden spike implying the presence of snap-back (note log scale on $y$-axis); (d) schematic of snap-back and energy dissipated by snap-through

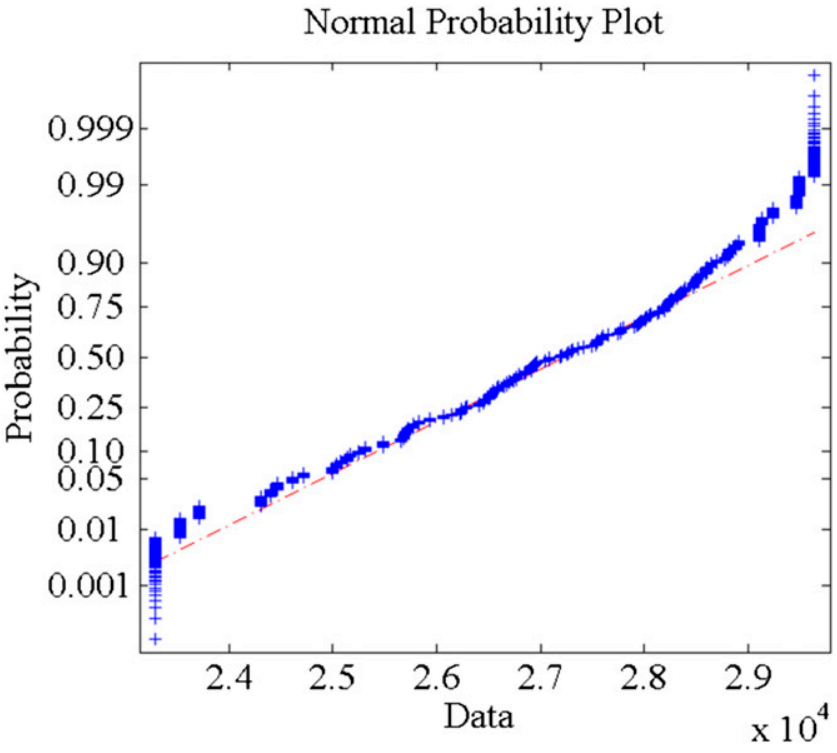

Fig. 9. Normal probability plot of the randomly generated values of the Young's modulus used in a simulation on the small-sized disk randomized material properties, the second fracture pattern seen in the tests could be reproduced for the small- and medium-sized disks. These results also suggest that the onset of postpeak instability with a sudden load drop may be related to the fracture patterns with one diametric crack or three radial cracks. However, for the largest size, a single diametrical crack was not obtained in the simulations. The random field of material properties provides initiation sites for the localization of damage (some material points being weaker than others). It appears that a random field with a much too small autocorrelation length cannot provide a potent enough initiation site for a single diametric crack.

\section{Autocorrelation Length}

The convenient assumption that the autocorrelation length is equal to the finite-element size (and the maximum aggregate size) is now relaxed. A three-dimensional Gaussian autocorrelated field of modulus $E$ is generated. The spectral method described by Cirpka (2003) is adopted to generate a field of random real numbers. In this method, the discrete Fourier transform of the covariance function (which describes the variance of a random field) is used to get the power spectral density (PSD) function of all realizations. Note that the PSD 


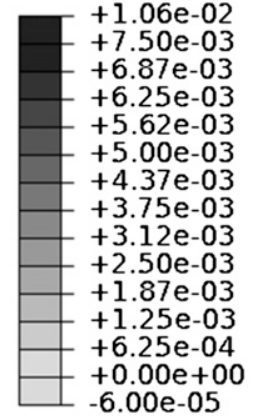

(a)

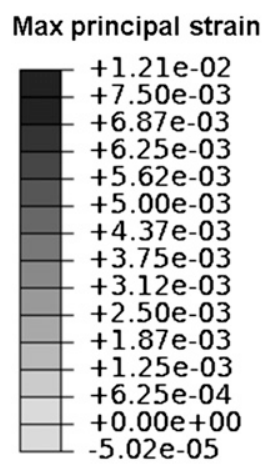

(b)

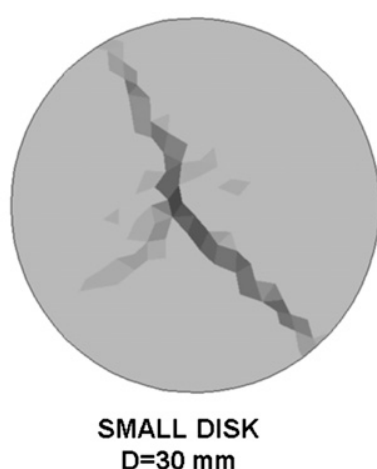

$\mathrm{D}=30 \mathrm{~mm}$

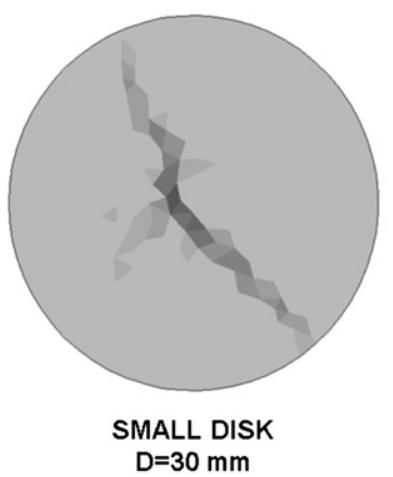

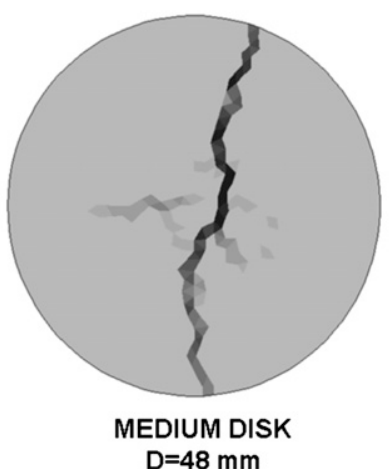

$D=48 \mathrm{~mm}$

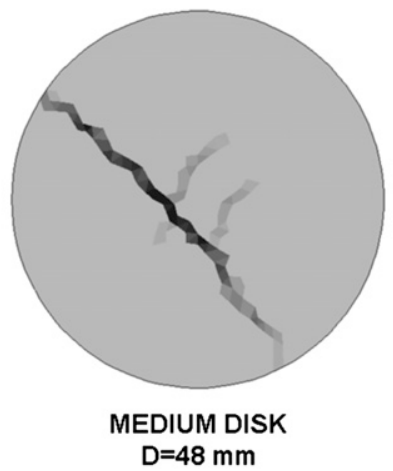

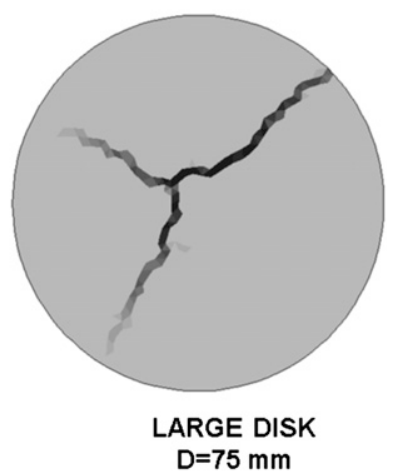

$D=75 \mathrm{~mm}$

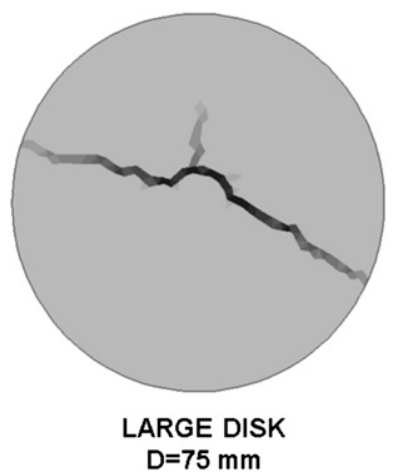

Fig. 10. (a) Predicted fracture patterns using a realistic test setup and a random field of Young's modulus values; (b) predicted fracture pattern using a realistic test setup and a random field of Young's modulus values with a high autocorrelation length

function describes the strength of the variations as a function of frequency. Random phase spectra, which are generated along with the power spectra, are backtransformed into the physical domain to yield the random autocorrelated fields. These fields satisfy the conditions specified for the mean, SD, and autocorrelation length within the specified physical domain and the mesh density used. The realization is incorporated into the finite-element model through the user's material subroutine VUMAT for explicit integration in ABAQUS.

Simulations are then run with random fields of different autocorrelation lengths with several simulations for each size. Thus it is empirically found that a good agreement with the tests is obtained for an autocorrelation length equal to four times the assumed crack bandwidth [which is equal to $52 \mathrm{~mm}$ (2.047 in.)]. The corresponding fracture pattern, shown in Fig. 10(b), consists of a single dominant diametric crack, which forms at the onset of postpeak instability for all three sizes. This is in agreement with the tests and confirms that autocorrelation is a necessary assumption.

The fracture patterns shown were obtained with a set of parameters that had initially been calibrated by fitting the test data from only one disk for each size. Subsequently, the parameters were recalibrated to fit the mean response from all the disks for each size, and it was verified by several runs that recalibration did not appreciably affect the fracture patterns.

Noting that Voronoi tessellation minimizes the bias of the fracture orientation, it transpires that upon taking into account the autocorrelation of the random $E$ modulus and strength, the microplane model can reproduce the fracture patterns observed on the disks of all three sizes, their failure loads, the size effect, and the load-deflection curves. This finding confirms that the plot of the flexural strength logarithm versus the disk size logarithm must terminate with the
Weibull statistical size effect, which is represented by an asymptote of a small downward slope equal to $-n / m$ where $m$ is the Weibull modulus (Bažant and Novák 2000) (about 24) and $n=3$ is the number of spatial dimensions. However, the sizes of the tested disks were not large enough reveal this asymptote.

\section{Remark on Postpeak Responses for Various Moduli of the Loading Ring}

Although this study is focused on the disk strength (or peak load) for which the postpeak response is irrelevant, it may nevertheless be of interest for future studies to comment on the postpeak. The small-size disk was simulated considering four cases of loading ring moduli, namely $500 \mathrm{MPa}-500 \mathrm{GPa}(72.5,725,7,250$, and $72,500 \mathrm{ksi})$, whereas the support ring modulus was maintained constant at $50 \mathrm{MPa}(7.25 \mathrm{ksi})$. The simulated fracture patterns are shown in Fig. 11 for these four cases. For the first two cases [Fig. 11, two top images], which have lower modulus values, the disks show a greater tendency to snap-through and fail with the three dominant radial cracks. This is similar to the previous case where the loading ring modulus was $50 \mathrm{MPa}(7.25 \mathrm{ksi})$. For the latter two cases [Fig. 11, two bottom images] with higher modulus values, the behavior changes. Here, there is no snap-through instability, the postpeak is stable, and multiple $(>3)$ radial cracks develop. Evidently, the fracture pattern and the postpeak instability with sudden load drop (which imply the presence of a snap-back) are related phenomena.

\section{Conclusions}

1. The biaxial flexure test has been simulated using a calibrated and validated microplane Model M7 on concrete disks of three 
Max principal strain

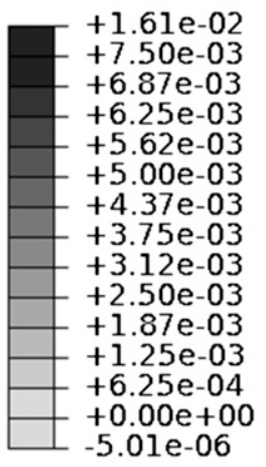

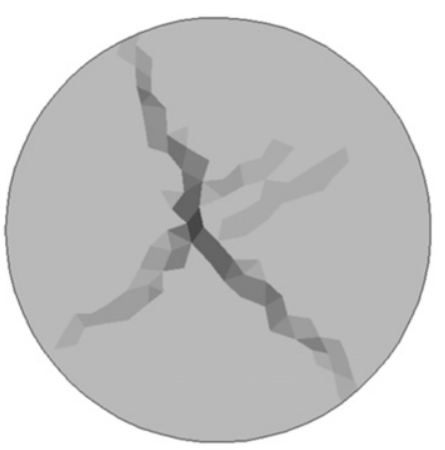
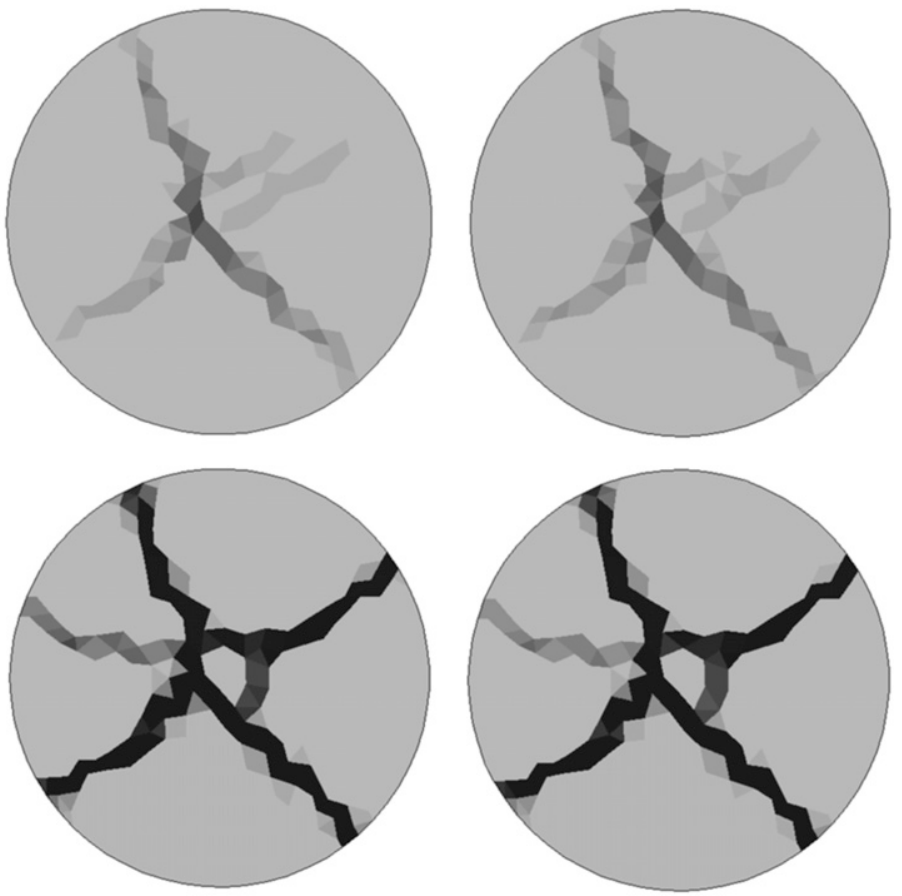

SMALL DISK

$\mathrm{D}=30 \mathrm{~mm}$

Fig. 11. Predicted fracture patterns on the small-size disk with varying modulus of the loading ring

sizes. The predictions of the model are found to be in good agreement with the test data.

2. The microplane model predicts both the uniaxial and biaxial size effects satisfactorily. Numerical simulations over a greatly extended size range indicate that the size effect is Type I, which characterizes failures that occur while the macrocrack initiates from a smooth surface.

3. The biaxial tensile strength is found to be higher than the uniaxial tensile strength from the simulations.

4. With realistic boundary conditions and an autocorrelated field of random material properties, the fracture patterns observed in the tests are correctly reproduced for the disks of all sizes.

5. The boundary conditions of the biaxial flexure of disks simulation have a strong influence on the stability of the numerical procedure and on the resulting fracture pattern. When the loading and the support are applied to the disk via an elastomeric ring (which is relatively soft), it causes instability right after the peak. This results in either one diametric crack or three radial cracks, which is the same as observed in the tests. However, when the displacement boundary conditions are applied directly to the nodes of the disk, stable postpeak softening is observed. In that case one gets a different fracture pattern, which consists of multiple radial cracks. In other words, damage localization in one diametric zone or three radial zones appears to be associated with postpeak instability and sudden load drop whereas damage localization in multiple radial cracks is associated with stable postpeak softening.

6. The modulus of the loading ring is seen to determine whether dynamic snap through instability occurs after the peak load. A sufficiently stiff loading ring leads to stable postpeak softening with multiple radial cracks whereas a sufficiently compliant ring leads to postpeak instability with sudden load drop, which is characterized by either one diametric crack or three radial cracks.

7. With autocorrelated random fields of elastic modulus and strength, the simulations correctly predict one diametric crack for the largest test specimen. Without these autocorrelated random fields, only the fracture pattern with three radial cracks is predicted.

8. This study provides an additional confirmation that microplane Model M7 is a realistic model for quasi-brittle fracture with size effects.

\section{Acknowledgments}

Financial support from the DOT, provided through Grant No. 20778 from the Infrastructure Technology Institute of Northwestern University, is gratefully appreciated. Additional support for the analysis was provided by NSF Grant No. CMMI-1129449 to Northwestern University. Computer support by Quest, the high performance cluster supercomputer at Northwestern University, was indispensable. The work of the third author was partially supported by the National Research Foundation of Korea (Grant No. 2012R1A1B3004227) to Korea University.

\section{References}

ABAQUS. (2011). ABAQUS/explicit user's manual-ABAQUS version 6.11, Dassault Systèmes Simulia, Providence, RI.

Asaro, R. J., and Rice, J. R. (1977). "Strain localization in ductile single crystals." J. Mech. Phys. Solids, 25(5), 309-338.

ASTM. (2008). "Standard test method for flexural toughness of fiberreinforced concrete (using centrally-loaded round panel)." C1550, West Conshohocken, PA. 
Batdorf, S., and Budianski, B. (1949). "A mathematical theory of plasticity based on the concept of slip." Technical Note No. 1871, National Advisory Committee for Aeronautics, Washington, DC.

Batdorf, S. B., and Crose, J. G. (1974). "A statistical theory for the fracture of brittle structures subjected to polyaxial stress states.” J. Appl. Mech., 41(2), 459-465.

Bažant, Z. (1984). "Microplane model for strain-controlled inelastic behavior." C. S. Desai and R. H. Gallagher, eds., Chapter 3, Mechanics of engineering materials, Wiley, London, 45-59.

Bažant, Z. P., Caner, F. C., Carol, I., Adley, M. D., and Akers, S. A. (2000). "Microplane model M4 for concrete. I: Formulation with work-conjugate deviatoric stress." J. Eng. Mech., 10.1061/(ASCE)0733-9399(2000) 126:9(944), 944-953.

Bažant, Z. P., and Cedolin, L. (2010). Stability of structures: Elastic, inelastic, fracture and damage theories, 3rd Ed., World Scientific, London.

Bažant, Z. P., and Novák, D. (2000). "Probabilistic nonlocal theory for quasibrittle fracture initiation and size effect. I: Theory." J. Eng. Mech., 10.1061/(ASCE)0733-9399(2000)126:2(166), 166-174.

Bažant, Z. P., and Novák, D. (2001). "Proposal for standard test of modulus of rupture of concrete with its size dependence.” ACI Mater. J., 98(1), 79-87.

Bažant, Z. P., and Oh, B.-H. (1985). "Microplane model for progressive fracture of concrete and rock." J. Eng. Mech., 10.1061/(ASCE)07339399(1985)111:4(559), 559-582.

Bažant, Z. P., and Planas, J. (1998). Fracture and size effect: In concrete and other quasibrittle materials, CRC, New York.

Caner, F. C., and Bažant, Z. P. (2000). "Microplane model M4 for concrete. II: Algorithm and calibration." J. Eng. Mech., 10.1061/(ASCE)07339399(2000)126:9(954), 954-961.

Caner, F. C. and Bažant, Z. P. (2013). "Microplane model M7 for plain concrete. I: Formulation.” J. Eng. Mech., 10.1061/(ASCE)EM.19437889.0000570, 1714-1723.

Caner, F. C., Bažant, Z. P., Hoover, C., Waas, A., and Shahwan, K. (2011) "Microplane model for fracturing damage of tri-axially braided fiberpolymer composites." J. Eng. Mater. Technol., 133(2), 021024.

Červenka, J., Bažant, Z. P., and Wierer, M. (2005). "Equivalent localization element for crack band approach to mesh-sensitivity in microplane model.” Int. J. Numer. Methods Eng., 62(5), 700-726.
Cirpka, O. A. (2003). "Generation of random, autocorrelated, periodic fields." Scientific/Educational MATLAB Database, 〈http://m2matlabdb .ma.tum.de/download.jsp?MC_ID =6\&MP_ID $=31\rangle$ (Oct. 2012).

Cusatis, G., Beghini, H., and Bažant, Z. P. (2008). "Spectral stiffness microplane model for quasi-brittle composite laminates: I. Theory." J. Appl. Mech., 75(1), 1-9.

Engineering Toolbox. (2012). "Modulus of elasticity-Young modulus for some common materials." 〈http://www.engineeringtoolbox.com/ young-modulus-d_417.html $\rangle$ (Aug. 2012).

Fessler, H., and Fricker, D. C. (1984). "A theoretical analysis of the ring-onring loading disk test." J. Am. Ceram. Soc., 67(9), 582-588.

Giovan, M. N., and Sines, G. (1979). "Biaxial and uniaxial data for statistical comparisons of a ceramic's strength." J. Am. Ceram. Soc., 62(9-10), $510-515$.

Kim, J., Yi, C., and Zi, G. (2012). "Biaxial flexural strength of concrete by two different methods." Mag. Concr. Res., 64(1), 21-33.

Lee, S., Song, Y., and Han, S. (2004). "Biaxial behavior of plain concrete of nuclear containment building." Nucl. Eng. Des., 227(2),143-153.

Mindess, S., and Francis, Y. J. (1981). Concrete, Prentice Hall, Englewood Cliffs, NJ.

Morrell, R. (1998). Biaxial flexural strength testing of ceramic materials: Measurement good practice guide, No. 12, National Physical Laboratory, Middlesex, U.K.

Northwestern University Information Technology. (2012). "Quest." $\langle$ http:// www.it.northwestern.edu/ research/adv-research/hpc/quest/index.html $\rangle$ (Jun. 2012)

Shetty, D. K., Rosenfield, A. R., and Duckworth, W. H. (1983). "Crack branching in ceramic disks subjected to biaxial flexure." J. Am. Ceram. Soc., 66(1), C-10-C-12.

Taylor, G. I. (1938). "Plastic strain in metals." J. Inst. Met. London, 62, $307-324$.

Timoshenko, S. P., and Woinowsky-Krieger, S. (1959). Theory of plates and shells, 2nd Ed., McGraw Hill, New York.

Zi, G., Oh, H., and Park, S.-K. (2008). "A novel indirect tensile test method to measure the biaxial tensile strength of concretes and other quasibrittle materials." Cement Concr. Res., 38(6), 751-756.

Zi, J., Kim, J., and Bažant, Z. P. (2013). "Size effect on biaxial tensile strength of concrete." ACI Mater. J., in press. 